\title{
7 Motor und Turbo - Was ist der richtige Ansatz für eine IT der zwei Geschwindigkeiten?
}

\author{
Peter Gocke
}

Medizin braucht Daten - dieser Ausruf zieht sich wie ein roter Faden durch die allgegenwärtigen Diskussionen zur Digitalen Transformation im Gesundheitswesen. Die sich daran anschließenden Erörterungen führen dann oft zu Themen, die diese gewünschte Nutzung von Daten aktuell stark einschränken: Hier werden beispielsweise veraltete IT-Systeme, unternehmens-organisatorische Hindernisse oder der Datenschutz genannt - Themen, die in anderen Kapiteln dieses Buches beleuchtet werden.

\section{Medizin braucht Daten!}

Wer treibt eigentlich die Digitale Transformation im Gesundheitswesen voran? Oftmals wird diese Aufgabe einfach bei der IT-Abteilung verortet und sodann die IT-Abteilung für die aus Unternehmens- und Anwendersicht zu langsam und ineffizient erfolgende Digitale Transformation verantwortlich gemacht. Aber ist das wirklich richtig?

In der Tat wurde in der Vergangenheit insbesondere im Gesundheitswesen ein Ansatz verfolgt, sich über die Beschaffung und Implementierung von IT-Systemen zu ,digitalisieren“. Letztlich wurde damit aber eher eine „Elektronifizierung“ erreicht, aber eben keine „Digitalisierung“ - und schon gar keine Digitale Transformation. Als Nachwehen dieser Ära sind jetzt aber vielerorts hauptsächlich IT-Abteilungen damit beschäftigt, das hierdurch entstandene Portfolio an unterschiedlichen IT-Systemen $z u$ betreiben und durch mehr oder weniger funktionierende Schnittstellen auch Prozesse zu unterstützen, die über die Abteilungsgrenzen dieser Systeme hinausgehen. 


\section{Elektronifizierung ist keine Digitalisierung!}

Hauptsächlich fehlt in diesen Szenarien genau die Unterstützung der Abteilungs- und teilweise sogar Unternehmensgrenzen überschreitender Prozesse: Die Systeme wurden eingeführt und an existierende Prozesse mehr oder weniger angepasst, es wurde aber nicht auf Basis einer übergreifenden Prozesslandschaft die dafür benötigte ITUnterstützung konzipiert: „process limits IT“ anstelle von „IT follows process“. Wird es dadurch oftmals schon schwierig, einen Patienten nur über eine rein elektronische Dokumentation von einer Abteilung eines Krankenhauses in eine andere zu verlegen, so basiert spätestens die Verlegung in ein anderes Krankenhaus auf einer papiergebundenen Dokumentation. Bedenkt man darüber hinaus, dass digitale Services z.B. bei der Vereinbarung von Arztterminen oder dem Anfordern von Verlängerungsrezepten von immer mehr Menschen nachgefragt werden, wird deutlich, dass die Digitalisierung des Gesundheitswesens erst in den Anfängen steckt.

\section{Digitale Transformation bedeutet: IT follows process!}

Wenn also auch IT-Abteilungen nicht hauptverantwortlich für die Digitale Transformation sind, so sind sie und die von diesen gelieferten Services selbstverständlich von großer Bedeutung für die Digitale Transformation von Unternehmen - und dies gilt letztlich auch für Krankenhäuser, Kliniken und Arztpraxen. Schaut man aber auf die Struktur einer „klassischen“ IT-Abteilung, so finden sich dort durchaus Ursachen für die zu Recht als zu langsam empfundene Digitale Transformation. Die Entwicklung der klassischen IT-Abteilung wurde letztlich von den IT-Bedürfnissen aus Verwaltung und Krankenversorgung getrieben, wo hohe Verfügbarkeit und Standardisierung für eine kontinuierlich sichere und kostengünstige Technik gefordert waren:

1. IT Services: Sicherheit und Verfügbarkeit stehen hier im Vordergrund - möglichst abgesichert durch strenge Service Level Agreements (SLAs).

2. IT Governance: Ein definiertes, wenig flexibles Portfolio von IT-Systemen soll sowohl sicherstellen, dass die Kosten im Rahmen bleiben, als auch einen stabilen Betrieb ermöglichen.

3. IT Prozesse: Klassische langwierige Prozesse (Wasserfall-Vorgehen) sollen Risiken bei der Einführung von Software minimieren - führen aber bei komplexen Projekten aufgrund der dann drastisch sinkenden Implementierungsgeschwindigkeit dazu, dass an anderen Stellen Risiken auftreten, beispielsweise durch langfristig bestehende Medienbrüche. Letztlich steigt auch das Risiko, dass noch zur Laufzeit des Projektes die eingesetzte Lösung überaltert! IT wird mehr verwaltet als gestaltet - auch das drückt sich in vielfach zu komplexen Beantragungs- und Genehmigungsprozessen aus.

4. Sourcing: Aus Kostensicht werden langfristige Verträge mit möglichst wenigen Partnern bevorzugt. Hierdurch steigt aber einerseits das Risiko von Abhängigkeiten, andererseits ist es mit einer solchen Ausrichtung der IT kaum möglich, von innovativen Ideen von Start-ups zu profitieren. 
5. Infrastruktur: Um die einmal getätigten Investitionen in Infrastruktur zu schützen, wird an diesen Systemen sehr lange festgehalten - und das Gefühl, alles selbst machen zu müssen, trägt ebenfalls dazu bei, dass neuere Techniken (Software as a Service, SaaS) keinen Platz im Portfolio finden. Gerade in diesen Bereichen erfolgen die Innovationen mittlerweile so schnell, dass auf veraltenden Plattformen oft keine adäquate Weiterentwicklung mehr erfolgen kann.

6. Management: Im Management, insbesondere der Krankenhaus-IT, herrschen oft noch klassische Rollenmodelle mit ausgeprägten Hierarchien vor. Dies stört in einer „langsamen“ IT nicht, wohl aber wenn agile Mitarbeiter und junge, eher experimentierfreudige Mitarbeiter für die aktuell anstehenden Anforderungen gesucht werden: Diese fühlen sich in Firmen mit flacheren Hierarchien deutlich wohler und entfalten dort auch eher ihr kreatives Potenzial

In Zeiten der Digitalen Transformation kommen aber weitere Anforderungen aus dem Unternehmen, insbesondere aus den Bereichen Forschung und Lehre, aber auch aus den Bedürfnissen der Patienten hinzu. Hier werden eher kunden- und lösungsorientierte Ansätze benötigt und weniger auf die Einhaltung von SLAs fokussiert. Mehr Flexibilität und eine höhere Umsetzungsgeschwindigkeit sollen sicherstellen, dass auch kurzfristigen Kundenwünschen adäquat begegnet werden kann. Neue Technologien sollen schneller genutzt werden, als eine „klassische“ IT sich diese zu Eigen machen kann: Hier sind auch Servicemodelle gefragt - selbstverständlich immer unter der Steuerung durch die hauseigenen IT-Strukturen. Letztlich geht es gerade in Zeiten des Fachkräftemangels auch darum, als Arbeitgeber attraktiv für IT-Mitarbeiter zu sein - dies erfordert ein Betriebsklima, in welchem Teamgedanken im Vordergrund stehen und selbstorganisierende Strukturen möglich sind. Was bedeutet das für die IT-Abteilung? Auch die IT-Abteilung steht vor einer Transformation!

\section{Auch IT-Abteilungen müssen transformiert werden.}

Abbildung 1 zeigt die Gegenüberdarstellung von „klassischer IT“ und „fast IT“. Selbstverständlich macht es keinen Sinn, zwei IT-Abteilung innerhalb eines Unternehmens zu betreiben - und auch die Ausprägung der IT zwischen den Polen „klassisch“ und „fast“ wird von Unternehmen zu Unternehmen unterschiedlich sein, je nach Bedürfnis und nach dem aktuell erreichten Digitalisierungsgrad. Insofern wurde der Begriff der „Right Speed IT“ geprägt, die einen flexiblen Übergang von der klassischen IT zur möglichst agilen IT ermöglichen soll, und dabei sicherstellen soll, dass eben keine zusätzliche IT-Struktur entsteht, sondern die IT vielmehr integraler Bestandteil des Unternehmens wird.

Die Unternehmenskultur und insbesondere die Fähigkeit eines Unternehmens, die IT-Strukturen tief in die Gesamtorganisation zu intergieren, hat großen Einfluss auch auf die Struktur der IT-Abteilung und den Erfolg bei der Digitalen Transformation. Die Digitale Transformation ist ein Unternehmensprojekt, kein IT-Projekt!

\section{Die Digitale Transformation ist ein Krankenhaus-Projekt, kein IT-Projekt.}




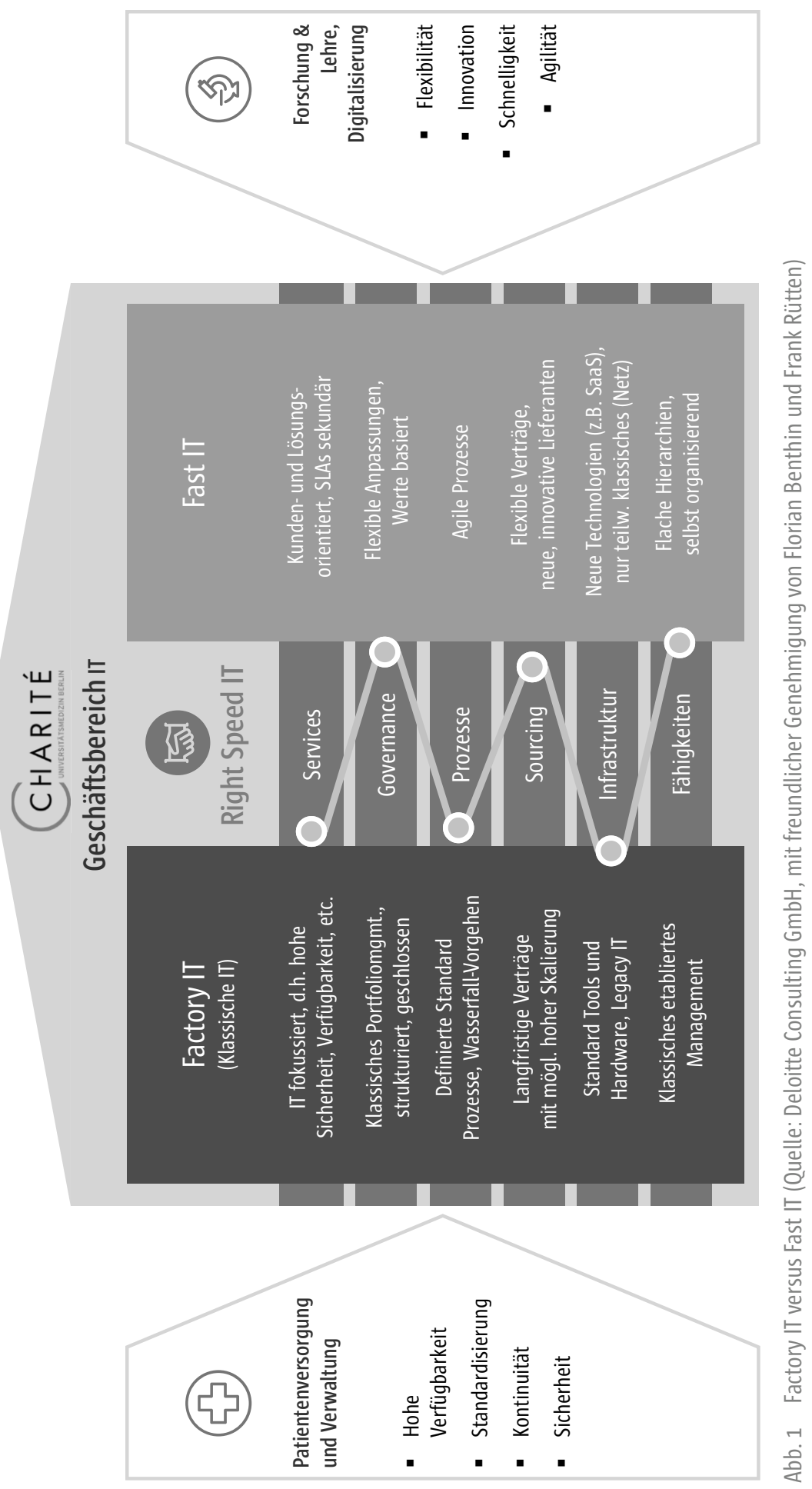


Das hat Auswirkungen auf die Organisation des gesamten Unternehmens. So macht es keinen Sinn, das Projektmanagement für die (oftmals nur sogenannten) „IT-Projekte“ von IT-Mitarbeitern mit übernehmen zu lassen. Dies belastet unnötig Ressourcen der IT-Abteilungen, in denen oftmals auch die benötigten Kenntnisse und Fähigkeiten für ein effizientes Projektmanagement gar nicht vorhanden sind. Insbesondere mit Blick auf eine echte Digitale Transformation gehören solche Projekte zentral gemanaged - und von zentralen oder dezentralen Projektmanagern geleitet. Bewährt hat sich hier der Aufbau eines Project Management Offices (PMO), bei welchem Projekte zentral angemeldet und nach Priorisierung und Entscheidung durch den Vorstand immer mit Blick auf die Unternehmensstrategie und Agenda der Digitalen Transformation umgesetzt werden. Allein der dadurch geförderte Aufbau von Projektmanagementwissen und Fähigkeiten erhöht die Umsetzungsgeschwindigkeit von Projekten und beschleunigt damit auch die Digitale Transformation.

Eine ähnlich klare Strukturierung benötigen auch andere mit der Digitalen Transformation assoziierten Themen, wie zum Beispiel Schulungen von Mitarbeitern, insbesondere für die implementierten Software-Systeme, aber auch die dabei eingeführten Prozesse. Oft wurde auch diese Aufgabe einfach der IT übertragen - mit den bereits beschriebenen Konsequenzen für Ressourcen-Fehlallokation und sinkender Umsetzungsgeschwindigkeit. Wenn sich dabei noch herausstellt, dass die in Schulungen aufkommenden Fragen oftmals mehr den Prozess als das Prozesswerkzeug (IT) betreffen, wird deutlich, dass es auch hierfür zentral zu organisierende Strukturen geben sollte.

Diese Aufzählungen lassen sich noch weiter fortsetzen: Ob es Themen des Datenschutzes oder der Berechtigungen sind, oftmals sind eigentlich andere Abteilungen im Unternehmen als die IT für diese Prozesse und Themen verantwortlich, und diese Verantwortung wechselt nicht dadurch in den Bereich der IT, weil das neue Prozesswerkzeug von der IT implementiert wird.

\section{Verantwortungen für einen Prozess ändern sich nicht durch ein neues Prozesswerkzeug.}

Nur Krankenhäuser und Unternehmen, die es schaffen, diese Integration der IT-Abteilungen in ihre Unternehmensstruktur zu vollziehen und eine „digitale Kultur“ zu etablieren, werden auf dem Weg zur Digitalen Transformation erfolgreich sein. Der Einsatz Digitaler Techniken darf niemals ein Selbstzweck werden, sondern muss immer an den Bedürfnissen eines Unternehmens und dem Dienst an dessen Kunden ausgerichtet sein. Die organisatorische Herausforderung liegt darin, einen Ausgleich zwischen den klassischen und den zukünftigen Strukturen zu gewährleisten: Transformation statt Disruption. 